\title{
BMJ Open Which factors influence decisions to transfer and treat patients with acute intracerebral haemorrhage and which are associated with prognosis? A retrospective cohort study
}

\author{
Kamran A Abid, ${ }^{1,2}$ Andy Vail, ${ }^{3}$ Hiren C Patel, ${ }^{1,4}$ Andrew T King, ${ }^{1,4}$ Pippa J Tyrrell,,${ }^{4,5}$ \\ Adrian R Parry-Jones ${ }^{4,5}$
}

To cite: Abid KA, Vail A, Patel HC, et al. Which factors influence decisions to transfer and treat patients with acute intracerebral haemorrhage and which are associated with prognosis? A retrospective cohort study. BMJ Open 2013;3:e003684. doi:10.1136/bmjopen-2013003684

- Prepublication history for this paper is available online. To view these files please visit the journal online (http://dx.doi.org/10.1136/ bmjopen-2013-003684).

Received 30 July 2013 Revised 4 October 2013 Accepted 4 November 2013

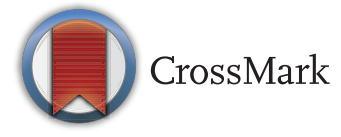

For numbered affiliations see end of article

Correspondence to Dr A R Parry-Jones; adrian.parry-jones@ manchester.ac.uk

\section{ABSTRACT}

Objectives: To identify factors associated with the decision to transfer and/or operate on patients with intracerebral haemorrhage (ICH) at a UK regional neurosurgical centre and test whether these decisions were associated with patient survival.

Design: Retrospective cohort study.

Setting: 14 acute and specialist hospitals served by the neurosurgical unit at Salford Royal NHS Foundation Trust, Salford, UK.

Participants: All patients referred acutely to neurosurgery from January 2008 to October 2010.

Outcome measures: Primary outcome was survival and secondary outcomes were transfer to the neurosurgical centre and acute neurosurgery.

Results: We obtained clinical data from 1364 consecutive spontaneous patients with ICH and 1175 cases were included in the final analysis. $140(12 \%)$ patients were transferred and $75(6 \%)$ had surgery. In a multifactorial analysis, the decision to transfer was more likely with younger age, women, brainstem and cerebellar location and larger haematomas. Risk of death in the following year was higher with advancing age, lower Glasgow Coma Scale, larger haematomas, brainstem ICH and intraventricular haemorrhage. The transferred patients had a lower risk of death relative to those remaining at the referring centre whether they had surgery (HR $0.46,95 \% \mathrm{Cl} 0.32$ to 0.67 ) or not (HR $0.41,95 \% \mathrm{Cl} 0.22$ to 0.73 ). Acute management decisions were included in the regression model for the 227 patients under either stroke medicine or neurosurgery at the neurosurgical centre and early do-not-resuscitate orders accounted for much of the observed difference, independently associated with an increased risk of death (HR 4.8, $95 \% \mathrm{Cl} 2.7$ to 8.6 ).

Conclusions: The clear association between transfer to a specialist centre and survival, independent of established prognostic factors, suggests aggressive supportive care at a specialist centre may improve survival in $\mathrm{ICH}$ and warrants further investigation in prospective studies.
Strengths and limitations of this study

- This study benefits from a large cohort of over a thousand patients with complete survival data likely to be representative of routine clinical practice in similar population centres in the UK.

- Although we have sought to adjust for all known predictors of survival after intracerebral haemorrhage, our retrospective study design could not determine causation.

- We did not have functional outcomes for our study cohort and further work is needed to investigate associations between transfer to neurosurgical care and long-term disability.

\section{INTRODUCTION}

Spontaneous intracerebral haemorrhage (ICH) represents a major worldwide health problem $^{12}$ and is responsible for $10-15 \%$ of all strokes. ICH is associated with the highest mortality and morbidity of all stroke subtypes and case fatality rate has remained unchanged for the past three decades. ${ }^{1} 3$ Although a number of large trials testing new therapies for ICH are underway, at present, there is no proven treatment for this condition and care remains largely supportive.

There has been increasing interest in admitting patients directly to specialist centres to improve outcomes in a diverse range of conditions including trauma, head injury and subarachnoid haemorrhage. ${ }^{4-6}$ A recent study of 205 patients with $\mathrm{ICH}$ presenting to emergency departments in non-tertiary hospitals in the USA found a non-significant trend towards reduced mortality with transfer to a tertiary care centre. ${ }^{7}$ Another US study reported lower mortality for patients with ICH treated in a neurological intensive care unit (ICU) rather than 
in a general ICU, but did not adjust for imaging findings and had missing premorbid health data for over half of the patients included in the study. ${ }^{8}$ These findings suggest that outcomes after ICH may be improved by timely and comprehensive supportive care at specialist centres, but this has not been tested in a large population in a healthcare system outside the USA.

In the UK, ICH is initially managed by physicians from diverse specialties including stroke medicine, emergency medicine and elderly care. Neurosurgical and neurocritical care expertise are typically concentrated in regional neurosciences centres such that the majority of patients are not admitted directly to hospitals with these services. Receiving hospitals typically refer most ICH cases to regional neurosurgical centres shortly after diagnosis. Although national guidelines have been published, ${ }^{9} 10$ there remains considerable uncertainty about the surgical management of $\mathrm{ICH}^{11}$ and practice is likely to vary.

We first examined the factors associated with the decisions to transfer and/or operate on patients with ICH referred acutely to neurosurgery in a large cohort of unselected patients with ICH representative of hospitalbased stroke care in the UK. Second, we sought to identify whether the decisions to transfer and/or operate on patients with ICH were associated with survival, independent of known prognostic factors.

\section{METHODS}

\section{Participants}

We included all patients referred to the Neurosurgical Department at Salford Royal NHS Foundation Trust (SRFT), Salford, UK with acute ICH from 14 regional hospitals between 1 January 2008 and 17 October 2010. Based on the reported incidence of ICH, ${ }^{1}$ it is likely that the majority (around $75 \%$ ) of new ICH cases in the catchment population of around 2.6 million were referred during the study period.

\section{Procedures}

Details of every referral made to the neurosurgical service are prospectively recorded by the duty neurosurgeon in an electronic referral database. The date and time of the referral, demographic details, history, examination (including the Glasgow Coma Scale (GCS) score at time of referral), investigation findings, diagnosis and the agreed management plan are recorded. Each case is reviewed by an on-call consultant neurosurgeon and consultant neuroradiologist within $24 \mathrm{~h}$. All ICH cases during our study period were reviewed by a study investigator and neurosurgeon (KAA) for inclusion in the study, blinded to survival status. Cases were excluded if there was clear evidence on the history of a traumatic cause for ICH, if the diagnosis was not ICH, if there were incomplete clinical data (including the GCS) or if the initial CT scan after presentation was not available for review. To provide a measure of premorbid health, the American Society of Anesthesiologists (ASA) grade $^{12}$ was determined based on details of comorbidities and premorbid functional status recorded in the neurosurgical database. A single study investigator (KAA) reviewed each patient's first CT scan and recorded haematoma location (deep, lobar, brainstem and cerebellum) and the presence or absence of hydrocephalus and intraventricular haemorrhage (IVH). Deep ICH was defined as involving deep supratentorial brain structures, including the basal ganglia and thalamus. Lobar ICH was defined as involving the cerebral lobes without involvement of deep structures. Brainstem location was defined as haemorrhage involving the medulla, pons and/or midbrain. Any haemorrhage within the cerebellum was defined as cerebellar. Where blood was evident in more than one compartment, the compartment containing the majority of the haematoma was recorded. Haematoma volume was determined by the $\mathrm{ABC} / 2$ method. ${ }^{13}$ For patients either initially presenting or transferred to SRFT, hospital records were reviewed to determine whether a 'do-not-resuscitate' (DNR) order was in place within $48 \mathrm{~h}$ of referral, their highest level of care during their acute admission (stroke/neurosurgical ward, Neurological High Dependency Unit (HDU) or ICU), whether further vascular imaging was performed, subsequent findings and whether any acute surgical intervention was performed. Once data collection and image analysis were complete, the survival status and date of death of non-survivors were obtained on 21 October 2011 via the Medical Research Information Service, Southport, UK which allowed a minimum follow-up period of 370 days for all patients.

\section{Statistical analysis}

Explanatory factors were log-transformed if positively skewed. A binary logistic regression model was used to investigate associations between decisions to transfer and operate on patients referred to the neurosurgical service. Cox regression analysis was performed to investigate factors associated with the risk of death during the 1-year period after referral.

In modelling, a rational selection procedure was applied to avoid overfitting where data were sparse, with factors exerting little influence on the outcome omitted from final models. Organisational factors (centre and consultant) were first screened in unifactorial analyses. If statistically significant they were included as strata when selecting clinical factors and retained only if they remained statistically significant after adjustment for retained clinical factors. For analysis of decision to operate, it was necessary to reduce the number of model parameters: centres were categorised according to their chance of having a transfer accepted; and ASA grades 3 and above were merged. For analysis of patient survival, follow-up time was censored beyond 1 year.

All analyses were performed with Stata (V.12, StataCorp LP, USA).

\section{Role of the funding source}

This research received no specific grant from any funding agency in the public, commercial or not-for- 
profit sectors. The employing institutions had no role in study design, in the collection, analysis and interpretation of data, in the writing of the report, or in the decision to submit the paper for publication. The corresponding author had full access to all study data and final responsibility for the decision to submit for publication.

\section{RESULTS}

In total, 1364 cases were coded as ICH in the referrals database for the period of our study and 189 were excluded for reasons outlined in figure 1. Of these, 1175 patients with complete data were included in the final analysis. The number of patients referred from each of the 14 hospitals ranged from 38 to 138, with the exception of one specialist oncology centre $(n=5)$ that does not receive acute patients directly. The baseline characteristics of the study population, categorised by whether they were transferred and had surgery, are outlined in table 1.

After adjustment for other variables, the decision to transfer a patient to the neurosurgical centre was associated with higher GCS score, brainstem and cerebellar location, larger haematomas, younger age and female sex (table 2). Higher GCS was predictive of transfer (OR

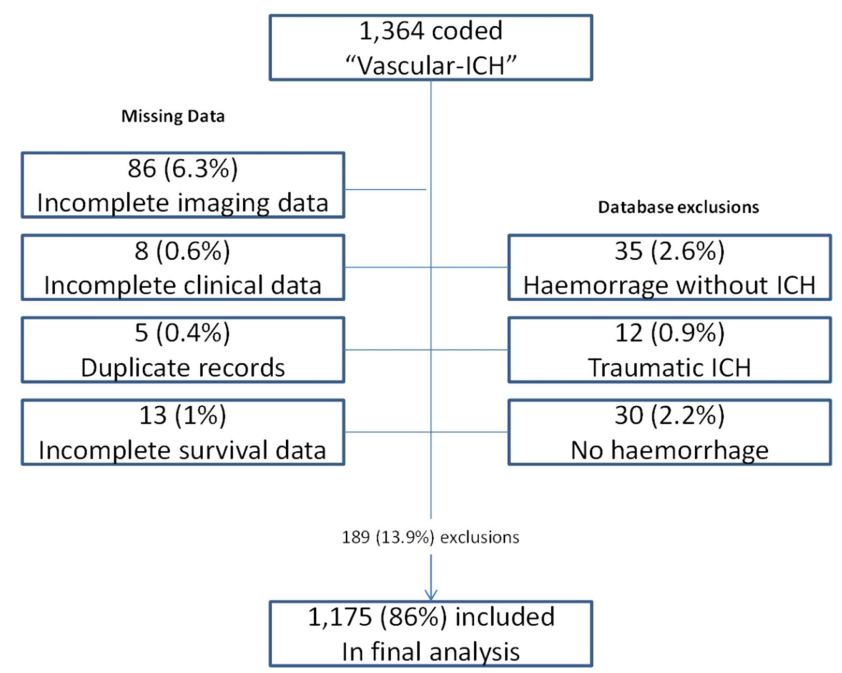

Figure 1 Study profile: 1364 cases were coded as 'Vascular-ICH' in the Neurosurgical referral database for the period of the study. Overall, 189 patients with incomplete data were excluded. Five duplicate entries were identified and a complete first brain CT scan was not available for 86 patients. Eight patients had missing clinical data. Thirty-five cases were excluded because they had intracranial haemorrhage without a component of $\mathrm{ICH}$ or had haemorrhagic transformation of an infarct. No haemorrhage was apparent on review of the CT scan for 30 patients. Twelve cases had a clear history of preceding trauma plus radiographic evidence of traumatic aetiology and were thus excluded. Survival data were obtained for all but 13 of the remaining patients, leaving 1175 $(86 \%)$ patients included in the final analysis. $\mathrm{ICH}$, intracerebral haemorrhage.
$1.13 ; 95 \%$ CI 1.06 to 1.21$)$. A strong association was found between infratentorial haemorrhage and transfer (OR 4.41; 95\% CI 2.31 to 8.40) and with each doubling in ICH volume the odds of transfer also approximately doubled. The odds of transfer more than halved with every 10 year increase in patient age (OR $0.41 ; 95 \%$ CI 0.35 to 0.48 ). Patients who had an ASA grade 3 (severe systemic disease) or more were less likely to be transferred than those who were previously healthy (ASA grade 1 ). There were differences in acceptance rates by referring centre. Post hoc rationalisation suggested that patients at designated stroke centres and those with greater travel times to the neurosurgical centre were less likely to be transferred. There was no evidence of differences in decision-making between the 19 consultant neurosurgeons. Hydrocephalus on the initial radiology report was excluded from the final model as there was no evidence of association with the decision to transfer.

Of the 140 patients transferred to neurosurgical care, 75 (54\%) had acute surgery. The decision to perform acute neurosurgery for the 140 transferred patients was associated with brainstem ICH location and lower GCS (table 3). Acute surgery was less common with lobar than deep location but this did not attain statistical significance. However, increasing haematoma volume was significantly associated with the decision to operate and examination of interaction terms in the model confirmed this was specifically for lobar haemorrhages. After taking in to account the GCS, location of haematoma and volume of haematoma, there was no evidence that the other variables (referring centre, responsible neurosurgeon, age, IVH, reported hydrocephalus on initial imaging or ASA grade) were associated with the decision to operate in this small dataset of 140 patients transferred to the neurosurgical centre.

One-year mortality for all patients was $53 \%$ and did not significantly differ between patients referred from the Greater Manchester Comprehensive Stroke Centre based at SRFT (54\%), all stroke centres combined $(52 \%)$ or all teaching hospitals combined $(53 \%)$, but was significantly lower in those transferred to neurosurgical care $(34 \%)$. In a multifactorial Cox regression analysis for the whole study population, increasing age, increasing haematoma volume, decreasing GCS, brainstem location, IVH and severe pre-existing patient comorbidity (ASA grade $\geq 3$ ) were found to be strongly associated with the hazard of death (table 4). After adjusting for these clinical prognostic factors, the decisions to transfer and operate remained strong predictors of survival (table 4, figure 2).

To further understand the reasons for improved survival for neurosurgical patients, we compared the acute management of patients who were under the care of the neurosurgical $(n=140)$ or stroke medicine $(n=87)$ teams at SRFT, as both groups were cared for at the same hospital with access to the same support facilities during the same time period. The differences between the groups are not significant, but neurosurgical patients tended to 
Table 1 Baseline characteristics of study population divided by transfer and surgery decisions

\begin{tabular}{|c|c|c|c|}
\hline & Not transferred $(n=1035)$ & $\begin{array}{l}\text { Transferred, no surgery } \\
(\mathrm{n}=65)\end{array}$ & Transferred, surgery $(n=75)$ \\
\hline \multicolumn{4}{|l|}{ Demographic } \\
\hline Age (years), median (IQR) & 75 (65 to 84$)$ & 56 (45 to 66$)$ & 55 (45 to 63$)$ \\
\hline Male, $\mathrm{n}(\%)$ & $546(52.8)$ & $34(52 \%)$ & $39(52 \%)$ \\
\hline Referring hospital, ID (n) & $\begin{array}{l}1(4), 2(94), 3(87), 4(78), \\
5(65), 6(47), 7(58), 8(43), \\
9(129), 10(88), 11(101), \\
12(33), 13(95), 14(113)\end{array}$ & $\begin{array}{l}1(1), 2(1), 3(2), 4(5), 5(5), \\
6(4), 7(4), 8(4), 9(4), \\
10(9), 11(11), 12(3), \\
13(5), 14(7)\end{array}$ & $\begin{array}{l}1(0), 2(2), 3(10), 4(0), \\
5(17), 6(8), 7(2), 8(4) \\
9(5), 10(6), 11(9), 12(2), \\
13(5), 14(5)\end{array}$ \\
\hline \multicolumn{4}{|l|}{ Clinical } \\
\hline Premorbid ASA grade (\%) & $\begin{array}{l}1(14 \%), 2(54 \%), 3(23 \%), \\
4 / 5(10 \%)\end{array}$ & $\begin{array}{l}1(31 \%), 2(58 \%), 3(8 \%), \\
4 / 5(3 \%)\end{array}$ & $\begin{array}{l}1(33 \%), 2(45 \%), 3(17 \%), \\
4 / 5(4 \%)\end{array}$ \\
\hline GCS, median (IQR) & 14 (7 to 15$)$ & $14(11$ to 15$)$ & 10 (7 to 13$)$ \\
\hline \multicolumn{4}{|l|}{ Imaging } \\
\hline $\begin{array}{l}\text { Haematoma volume }(\mathrm{mL}) \text {, } \\
\text { median (IQR) }\end{array}$ & 23.6 (6.8 to 63.4$)$ & 26.9 (12.6 to 50.4$)$ & 49.5 (23.2 to 87.6$)$ \\
\hline Deep, n (\%) & $444(43 \%)$ & $26(40 \%)$ & $33(44 \%)$ \\
\hline Lobar, n (\%) & $481(46 \%)$ & 31 (48\%) & 25 (33\%) \\
\hline Brainstem, n (\%) & $74(7 \%)$ & $1(2 \%)$ & $10(13 \%)$ \\
\hline Cerebellum, n (\%) & $36(3 \%)$ & $7(11 \%)$ & $7(9 \%)$ \\
\hline Hydrocephalus, n (\%) & $503(49 \%)$ & $23(35 \%)$ & $49(65 \%)$ \\
\hline $\begin{array}{c}\text { Intraventricular } \\
\text { haemorrhage, } \mathrm{n}(\%)\end{array}$ & $383(37 \%)$ & $19(29 \%)$ & $44(59 \%)$ \\
\hline
\end{tabular}

have larger haematomas, more IVH and a lower GCS, but younger age and a better premorbid ASA grade (table 5). We applied the Cox regression model to the subset of patients admitted to SRFT $(n=227)$ and estimated effects were broadly similar to the whole population, although with wider CIs reflecting the reduced sample size. This suggested that the SRFT stroke medicine patients were representative of those not transferred to neurosurgery (table 4).

Within the SRFT patients, marked differences between stroke medicine and neurosurgery were identified in the number of patients transferred to higher level care, the

Table 2 Predictors of transfer to neurosurgical care

\begin{tabular}{lllc}
\hline Factor & OR & 95\% Cl & p Value \\
\hline Age (per 10 year increase) & 0.41 & $(0.35$ to 0.48$)$ & $<0.001$ \\
Female & 1.92 & $(1.22$ to 3.01$)$ & 0.005 \\
GCS & 1.11 & $(1.05$ to 1.18) & 0.001 \\
ASA grade 2 (vs 1) & 0.78 & $(0.46$ to 1.34) & 0.37 \\
ASA grade 3 (vs 1) & 0.43 & $(0.21$ to 0.90) & 0.03 \\
ASA grade 4/5 (vs1) & 0.16 & $(0.05$ to 0.54) & 0.003 \\
log ICH volume & 1.97 & $(1.58$ to 2.45) & $<0.001$ \\
Lobar (vs deep) & 0.72 & $(0.43$ to 1.19) & 0.20 \\
Brainstem (vs deep) & 2.42 & $(1.00$ to 5.82) & 0.05 \\
Cerebellum (vs deep) & 6.37 & (2.65 to 15.29) & $<0.001$ \\
\hline 140 (12\%) patients were transferred acutely to neurosurgical care \\
and 1035 (88\%) remained at their referring hospital for further \\
care. Referring hospital was included in the regression model and \\
was significantly associated with the decision to transfer (p=0.02). \\
ASA, American Society of Anesthesiologists grade; GCS, \\
Glasgow Coma Scale; ICH, intracerebral haemorrhage.
\end{tabular}

use of DNR orders and whether further vascular imaging was performed (table 5). To determine whether these factors accounted for the improved outcomes seen in neurosurgical patients, the Cox regression was rerun from 24 to $48 \mathrm{~h}$ to include these factors, excluding patients who had died before each time point. Results are shown for $24 \mathrm{~h}$ as the differences were apparent by this time. Hazard of death was higher for those with DNR decisions in place and lower in those who underwent vascular imaging, regardless of the findings (normal, arteriovenous malformation (AVM) or aneurysm). There was no evidence of association with the acute care setting. After adjustment for these

Table 3 Predictors of surgical treatment in transferred patients

\begin{tabular}{lclc}
\hline Factor & OR & $\mathbf{9 5 \%}$ Cl & p Value \\
\hline GCS & $0.77(0.68$ to 0.87$)$ & $<0.001$ \\
log ICH volume & $1.49(1.002$ to 2.21$)$ & 0.049 \\
Lobar ICH (vs deep) & $0.34(0.15$ to 0.77$)$ & 0.009 \\
Infratentorial (vs & $4.45(1.40$ to 14.12$)$ & 0.011 \\
supratentorial) & & & \\
GCS & $0.77(0.68$ to 0.88$)$ & $<0.001$ \\
log ICH volume & $1.54(1.03$ to 2.30$)$ & 0.04 \\
Lobar (vs deep) & $0.44(0.19$ to 1.03$)$ & 0.06 \\
Brainstem (vs deep) & $18.2(1.72$ to 192.8) & 0.02 \\
Cerebellum (vs deep) & $1.03(0.27$ to 4.01$)$ & 0.96 \\
\hline
\end{tabular}

Of the 140 patients transferred to neurosurgical care, 75 underwent surgery.

GCS, Glasgow Coma Scale; ICH, intracerebral haemorrhage. 
Table 4 Factors predicting risk of death in 1-year follow-up period

\begin{tabular}{|c|c|c|c|c|c|c|}
\hline \multirow[b]{2}{*}{ Factor } & \multicolumn{3}{|c|}{ Whole population $(n=1175)$} & \multicolumn{3}{|c|}{ SRFT patients only $(n=227)$} \\
\hline & HR & $95 \% \mathrm{Cl}$ & p Value & HR & $95 \% \mathrm{Cl}$ & p Value \\
\hline Age (per 10 year increase) & 1.17 & (1.10 to 1.25$)$ & $<0.001$ & 1.27 & (1.07 to 1.51$)$ & 0.006 \\
\hline GCS & 0.81 & (0.79 to 0.83 ) & $<0.001$ & 0.80 & (0.75 to 0.85$)$ & $<0.001$ \\
\hline ASA grade $=2($ vs 1$)$ & 1.16 & (0.89 to 1.51$)$ & 0.28 & 2.22 & (1.18 to 4.20$)$ & 0.01 \\
\hline ASA grade $\geq 3($ vs 1$)$ & 1.63 & (1.24 to 2.14 ) & $<0.001$ & 2.12 & (1.09 to 4.13$)$ & 0.03 \\
\hline log $\mathrm{ICH}$ volume & 1.30 & (1.18 to 1.38$)$ & $<0.001$ & 1.13 & (0.90 to 1.41$)$ & 0.29 \\
\hline Lobar (vs deep) & 0.99 & (0.82 to 1.18$)$ & 0.88 & 1.39 & (0.87 to 2.23 ) & 0.17 \\
\hline Brainstem (vs deep) & 1.82 & (1.35 to 2.45$)$ & $<0.001$ & 2.75 & (1.35 to 5.64$)$ & 0.006 \\
\hline Cerebellum (vs deep) & 1.46 & (0.96 to 2.22$)$ & 0.07 & 1.09 & (0.44 to 2.68$)$ & 0.85 \\
\hline IVH & 1.54 & (1.28 to 1.84$)$ & $<0.001$ & 1.55 & (0.96 to 2.52$)$ & 0.08 \\
\hline Neurosurgical care, no surgery* & 0.41 & (0.22 to 0.73$)$ & 0.003 & 0.39 & (0.19 to 0.79$)$ & 0.01 \\
\hline Neurosurgical care, surgery* & 0.46 & (0.32 to 0.67$)$ & $<0.001$ & 0.53 & (0.30 to 0.94$)$ & 0.03 \\
\hline
\end{tabular}

${ }^{*}$ Compared with remaining at referring location for further care.

ASA, American Society of Anesthesiologists grade; GCS, Glasgow Coma Scale; ICH, intracerebral haemorrhage; IVH, intraventricular haemorrhage; SRFT, Salford Royal NHS Foundation Trust.

associations, the effect of transfer to neurosurgery on survival was lost (table 6). For the 139 (61\%) patients without a DNR order, there were only two calls to the cardiac arrest team. The first was due a respiratory periarrest and the second was the result of a seizure without cardiac or respiratory arrest, and both patients survived.

\section{DISCUSSION}

In our large UK cohort of patients with ICH, the decision to transfer to our neurosurgical unit was more likely in those with infratentorial ICH, higher GCS, younger age, larger haematomas and female sex. For transferred patients, the hazard of death, adjusted for established ICH prognostic factors, was less than half that of those remaining at their referring hospital.

Our study has a number of strengths. We have studied a large cohort of over a thousand patients with complete

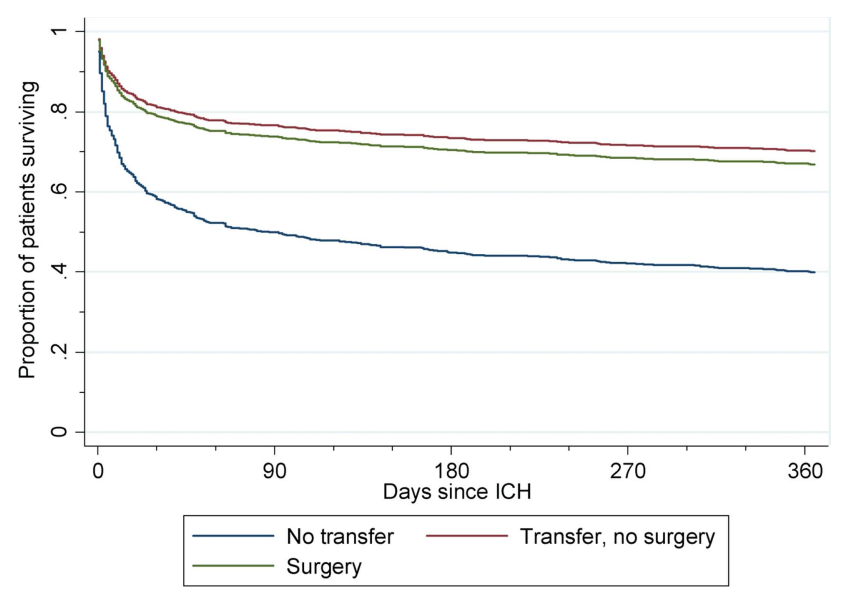

Figure 2 Kaplan-Meier survival curve for different management groups adjusted for case mix. $\mathrm{ICH}$, intracerebral haemorrhage; transferred, no surgery, $n=65 / 1175$ (6\%); transferred, surgery, $n=75 / 1175(6 \%)$; not transferred, $n=1035 /$ 1175 (88\%). survival data likely to be representative of routine clinical practice in similar population centres in the UK. All clinical data were prospectively recorded in the electronic neurosurgical database by a neurosurgeon at the time of referral and each case was reviewed by a senior neurosurgeon and neuroradiologist within $24 \mathrm{~h}$ of referral. Therefore, accurate and complete clinical information was available for the great majority of patients with ICH referred during the study period. Data collection and image analysis for this study were conducted by a single investigator, blinded to outcome, which minimises the introduction of bias. Our study also has some limitations; first, we excluded $107(8 \%)$ cases due to missing data although this was unlikely to have greatly affected our overall conclusion. Second, we have retrospectively estimated premorbid ASA grade from the information recorded in the neurosurgical database. Clinical information recorded in the neurosurgical database was fairly detailed for most cases, but it remains possible that the information was incomplete for some, thus underestimating the effect of premorbid health on mortality. Third, we believe that we have captured approximately $75 \%$ of the incident ICH cases in our population, but bias may have been introduced by case selection from referring hospitals. Fourth, because the study was limited to a single receiving centre, it is uncertain whether our findings are likely to be relevant to practice in other neurosurgical centres. However, the large sample size of our study and the fact that decisions were made by 1 of 19 consultant neurosurgeons increases the relevance of our conclusions to other centres and surgeons. Finally, functional outcome is of the utmost importance to patients and carers but we did not have these data in our ICH survivors. A previous study has shown worse 3-month cognitive outcomes in transferred versus non-transferred patients with ICH despite similar admission scores. ${ }^{14}$ It is not clear whether improved survival with neurosurgical care may be at the expense of an increased proportion of patients left alive but with 
Table 5 Clinical characteristics, management and survival at Salford Royal Hospital by responsible care team

\begin{tabular}{|c|c|c|}
\hline Factor & $\begin{array}{l}\text { Surgical } \\
(n=140)\end{array}$ & $\begin{array}{l}\text { Medical } \\
(n=87)\end{array}$ \\
\hline \multicolumn{3}{|l|}{ Demographic } \\
\hline Age (years), median (IQR) & 55 (45 to 65$)$ & $73(61$ to 81$)$ \\
\hline Male, n (\%) & $73(52 \%)$ & $53(61 \%)$ \\
\hline \multicolumn{3}{|l|}{ Clinical } \\
\hline \multirow[t]{4}{*}{ Premorbid ASA grade (\%) } & 1 (32\%), & $1(18 \%)$ \\
\hline & $2(51 \%)$ & $2(56 \%)$ \\
\hline & $3(13 \%)$ & $3(15 \%)$ \\
\hline & $4 / 5(4 \%)$ & $4 / 5(10 \%)$ \\
\hline GCS, median (IQR) & $12(8$ to 15$)$ & 14 (10 to 15$)$ \\
\hline \multicolumn{3}{|l|}{ Imaging } \\
\hline $\mathrm{ICH}$ volume $(\mathrm{mL})$, median & 36.1 & 25.4 \\
\hline (IQR) & (15.5 to 76.4$)$ & (9.1 to 64.9 ) \\
\hline Deep, n (\%) & $59(42 \%)$ & $45(52 \%)$ \\
\hline Lobar, n (\%) & $56(40 \%)$ & 27 (31\%) \\
\hline Brainstem, n (\%) & $11(8 \%)$ & $10(12 \%)$ \\
\hline Cerebellum, n (\%) & $14(10 \%)$ & $5(6 \%)$ \\
\hline Hydrocephalus, n (\%) & $72(51 \%)$ & $46(53 \%)$ \\
\hline $\mathrm{IVH}, \mathrm{n}(\%)$ & $63(45 \%)$ & $30(34 \%)$ \\
\hline \multicolumn{3}{|l|}{ Acute care setting, $\mathrm{n}(\%)$} \\
\hline ICU or NHDU within $24 \mathrm{~h}$ & $90(64 \%)$ & $9(10 \%)$ \\
\hline ICU or NHDU within $48 \mathrm{~h}$ & $92(66 \%)$ & $11(13 \%)$ \\
\hline \multicolumn{3}{|l|}{ Vascular imaging, $\mathrm{n}(\%)$} \\
\hline Within $24 \mathrm{~h}$ & $51(36 \%)$ & $6(7 \%)$ \\
\hline Within $48 \mathrm{~h}$ & $56(40 \%)$ & $7(8 \%)$ \\
\hline AVM identified & $13(9 \%)$ & $1(1 \%)$ \\
\hline Aneurysm identified & $20(14 \%)$ & $0(0 \%)$ \\
\hline \multicolumn{3}{|c|}{ Do not attempt resuscitation, n (\%) } \\
\hline Within $24 \mathrm{~h}$ & $31(22 \%)$ & $56(64 \%)$ \\
\hline Within $48 \mathrm{~h}$ & $32(23 \%)$ & $56(64 \%)$ \\
\hline \multicolumn{3}{|l|}{ Surgery, n (\%) } \\
\hline No surgery & $65(46 \%)$ & $87(100 \%)$ \\
\hline ICP monitor only & $2(1 \%)$ & NA \\
\hline EVD & $23(16 \%)$ & NA \\
\hline Haematoma evacuation & $50(36 \%)$ & NA \\
\hline Dead at 30 days, $n(\%)$ & $39(28 \%)$ & $38(44 \%)$ \\
\hline
\end{tabular}

ASA, American Society of Anesthesiologists grade; AVM, arteriovenous malformation; EVD, external ventricular drain; GCS, Glasgow Coma Scale; ICH, intracerebral haemorrhage; ICP, intracranial pressure; ICU, Intensive Care Unit; IVH, intraventricular haemorrhage; NHDU, Neurological High Dependency Unit.

severe long-term neurological deficits. Further prospective studies will be required to definitively answer this question.

Previous US studies have provided some evidence to support improved survival after ICH in those treated in specialist neurological ICUs (when compared with nonspecialist ICUs) and/or in tertiary centres. ${ }^{7} \quad 8 \quad 15$ Although better access to neurocritical care may lead to improved detection and treatment of the complications of ICH including acute hypertension, airway management, seizures, coagulopathy and hospital-acquired infections, ${ }^{16}$ we did not find transfer to ICU/HDU to be associated with improved survival after adjustment for other factors. However, transfer to ICU/HDU may be a marker of later neurological/general decline with an associated worsening of prognosis. We do not have information relating to neurological/general decline beyond the acute phase, so no clear conclusions can be drawn from this finding. ICHs caused by AVMs are associated with a lower mortality than ICH due to other causes ${ }^{17}$ but aneurysmal ICH carries a fairly poor prognosis. ${ }^{18}$ Patients with ICH in whom there are reasons to suspect an underlying AVM or aneurysm (imaging characteristics, age and/or history) are often transferred to neurosurgery, primarily for further vascular imaging. If patients with AVMs (but not aneurysms) are overrepresented, this may improve overall survival for those transferred to neurosurgical care and may account for the association seen in our study between performing further vascular imaging and improved survival. However, AVMs were found in $9 \%$ and aneurysms in $14 \%$ of neurosurgical patients and a systematic review of published studies found an overall incidence of $20 \%$ for AVMs and $13 \%$ for aneurysms. ${ }^{19}$ Although most studies have probably overestimated the incidence of vascular malformations due to selection bias, vascular malformations were found less often in our study than in previous published studies. We found no association with the findings of vascular imaging (when performed) and survival in our study. However, as most stroke medicine patients did not have vascular imaging, we cannot completely rule out the introduction of bias in our study from a differing mix of underlying aetiologies between the neurosurgery and stroke medicine patients.

The overall transfer rate to the neurosurgical unit was 12\% (140 patients) of ICH referrals. Despite contemporaneous national guidelines recommending initial medical management for infratentorial $\mathrm{ICH},{ }^{9}$ infratentorial location was strongly associated with the decision to transfer. The preference for transferring younger patients may partly be related to the increased likelihood of finding an underlying vascular malformation and hence a lower threshold for early transfer and investigation. Advancing age greatly reduced the likelihood of transfer and this seems to be independent of premorbid health. This may represent a reluctance to operate on older patients as there is evidence of worse outcomes from neurosurgery with older age in other conditions. ${ }^{20-22}$ Why female sex was positively associated with the decision to transfer is not clear, though this may relate to the higher incidence of cerebral aneurysms in women. ${ }^{23}$ Among transferred patients, the decision to intervene surgically was more likely with larger haematomas and infratentorial location, probably due to the propensity of infratentorial bleeds to cause herniation syndromes and/or obstructive hydrocephalus. Clearly, there are reasons why neurosurgeons may transfer patients with ICH to their care that will carry an associated survival benefit, independent of further care, which will introduce unmeasured confounding. Although younger patients with better premorbid health 
Table 6 Management factors within the first $24 \mathrm{~h}$ associated with risk of death in first year for SRFT patients

\begin{tabular}{|c|c|c|}
\hline Factor & Unadjusted* HR (95\% Cl) & Adjusted† HR (95\% Cl) \\
\hline \multicolumn{3}{|l|}{ Transfer decision } \\
\hline No transfer & 1.0 & 1.0 \\
\hline Transfer, no surgery & $0.46(0.22$ to 0.98$)$ & $1.3(0.53$ to 3.10$)$ \\
\hline Transfer and surgery & $0.68(0.35$ to 1.30$)$ & 1.60 (0.65 to 3.93$)$ \\
\hline \multicolumn{3}{|l|}{ Care setting } \\
\hline Ward (level 1) & 1.0 & 1.0 \\
\hline ICU/NHDU & $1.02(0.55$ to 1.87$)$ & 1.15 (0.53 to 2.50$)$ \\
\hline \multicolumn{3}{|l|}{ Vascular imaging } \\
\hline No & 1.0 & 1.0 \\
\hline Yes & $0.35(0.17$ to 0.74$)$ & $0.31(0.14$ to 0.68$)$ \\
\hline \multicolumn{3}{|l|}{ DNR } \\
\hline No & 1.0 & 1.0 \\
\hline Yes & 4.57 (2.70 to 7.73$)$ & $4.83(2.70$ to 8.64$)$ \\
\hline
\end{tabular}

were transferred to neurosurgery, transferred patients also tended to have larger haematomas and were more likely to have IVH. However, after adjusting for all established prognostic factors (including GCS, ICH volume, age, ICH location, IVH, hydrocephalus and premorbid health), neurosurgical care remained strongly associated with lower hazard of death. To further understand this finding, we compared patients within our study population who were under either neurosurgery or stroke medicine at the same hospital (SRFT). DNR orders were in place during the acute phase $(<48 \mathrm{~h})$ in a far higher proportion of stroke medicine patients and this was strongly and independently associated with a higher hazard of death. The use of early DNR orders has previously been shown to be independently associated with reduced survival after $\mathrm{ICH}^{24}$ so DNR orders within $48 \mathrm{~h}$ of admission are advised against in the recent US guidelines. $^{25}$ Although DNR orders should not limit other aspects of ICH care, they may subsequently lead to a less aggressive approach to care by the wider care team. Alternatively, they may be markers of less measurable but unalterable prognostic factors and our retrospective design could not determine causation. It may be that a more aggressive approach to supportive care after ICH in neurosurgical patients is responsible for improved survival and that the infrequent use of early DNR orders and high rate of vascular imaging are markers of this. In addition to the studies discussed above, further indirect evidence for the potential benefits of aggressive supportive care in ICH is provided by the surprisingly low mortality rates for patients recruited to the Clot Lysis: Evaluating Accelerated Resolution of Intraventricular Hemorrhage (CLEAR) II trial. Patients included in the trial would be expected to have a high mortality based on their clinical and imaging characteristics but had around 20\% mortality in the active and placebo groups. ${ }^{26}$ Further support for this theory is available for head injury, where observational data have supported improved outcomes with direct admission to a neurosurgical centre, ${ }^{4}$ and a randomised controlled trial to test this hypothesis is currently underway (ISRCTN68087745).

Our findings support the hypothesis that aggressive supportive care in specialist centres may improve survival after acute ICH and this warrants further investigation. This may have implications for the organisation of care for patients with ICH in the UK and internationally.

\section{Author affiliations}

${ }^{1}$ Department of Neurosurgery, Salford Royal NHS Foundation Trust, Salford, UK

${ }^{2}$ Faculty of Life Sciences, University of Manchester, Manchester, UK

${ }^{3}$ Health Sciences Research Group, University of Manchester, Manchester, UK

${ }^{4}$ The University of Manchester, Manchester Academic Health Sciences Centre, Salford Royal NHS Foundation Trust, Salford, UK

${ }^{5}$ Greater Manchester Comprehensive Stroke Centre, Salford Royal NHS Foundation Trust, Salford, UK

Contributors KAA contributed to the design of the study, collected the data, analysed CT scans and wrote the first draft of the manuscript. AV contributed to the design of the study, undertook the statistical analyses, and reviewed and contributed to the final draft of the manuscript. ATK, HCP and PJT contributed to the design of the study and reviewed the final draft of the manuscript. ARP-J contributed to the study design, had overall responsibility for the work, assisted with the data analysis and wrote the final draft of the manuscript and is the guarantor of the study. All authors, external and internal, had full access to all of the data (including statistical reports and tables) in the study and took responsibility for the integrity of the data and the accuracy of the data analysis.

Funding This research received no specific grant from any funding agency in the public, commercial or not-for-profit sectors.

Competing interests None.

Ethics approval Ethics approval was granted for this study by NHS Research Ethics Committee North West 10—GM North; Ref 11/H1011/3.

Provenance and peer review Not commissioned; externally peer reviewed.

Data sharing statement No additional data are available.

Open Access This is an Open Access article distributed in accordance with the Creative Commons Attribution Non Commercial (CC BY-NC 3.0) license, which permits others to distribute, remix, adapt, build upon this work noncommercially, and license their derivative works on different terms, provided the original work is properly cited and the use is non-commercial. See: http:// creativecommons.org/licenses/by-nc/3.0/ 


\section{REFERENCES}

1. Van Asch CJ, Luitse MJ, Rinkel GJ, et al. Incidence, case fatality, and functional outcome of intracerebral haemorrhage over time, according to age, sex, and ethnic origin: a systematic review and meta-analysis. Lancet Neurol 2010;9:167-76.

2. Qureshi Al, Mendelow AD, Hanley DF. Intracerebral haemorrhage. Lancet 2009;373:1632-44.

3. Béjot $\mathrm{Y}$, Cordonnier C, Durier J, et al. Intracerebral haemorrhage profiles are changing: results from the Dijon population-based study. Brain 2013;136:658-64.

4. Patel HC, Bouamra O, Woodford $\mathrm{M}$, et al. Trends in head injury outcome from 1989 to 2003 and the effect of neurosurgical care: an observational study. Lancet 2005;366:1538-44.

5. Poon WS, Li AK. Comparison of management outcome of primary and secondary referred patients with traumatic extradural haematoma in a neurosurgical unit. Injury 1991;22:323-5.

6. Bardach NS, Zhao S, Gress DR, et al. Association between subarachnoid hemorrhage outcomes and number of cases treated at California hospitals. Stroke 2002;33:1851-6.

7. Adeoye O, Haverbusch M, Woo D, et al. Is ED disposition associated with intracerebral hemorrhage mortality? Am J Emerg Med 2011;29:391-5

8. Diringer MN, Edwards DF. Admission to a neurologic/neurosurgical intensive care unit is associated with reduced mortality rate after intracerebral hemorrhage. Crit Care Med 2001;29:635-40.

9. Conditions NCGC for A and C, NICE. Stroke. 2008. http:// publications.nice.org.uk/stroke-cg68 (accessed 29 Mar 2012).

10. Intercollegiate Stroke Working Party. National clinical guideline for stroke, 4th edn. London: Royal College of Physicians, 2012.

11. Gregson BA, Broderick JP, Auer LM, et al. Individual patient data subgroup meta-analysis of surgery for spontaneous supratentorial intracerebral hemorrhage. Stroke 2012;43:1496-504.

12. Owens WD, Felts JA, Spitznagel EL Jr. ASA physical status classifications: a study of consistency of ratings. Anesthesiology 1978;49:239-43.

13. Kothari RU, Brott T, Broderick JP, et al. The ABCs of measuring intracerebral hemorrhage volumes. Stroke 1996;27:1304-5.

14. Catalano AR, Winn HR, Gordon E, et al. Impact of interhospital transfer on complications and outcome after intracranial hemorrhage. Neurocrit Care 2012;17:324-33.
15. Naval NS, Carhuapoma JR. Impact of pattern of admission on ICH outcomes. Neurocrit Care 2010;12:149-54.

16. Rincon F, Mayer SA, Rivolta J, et al. Impact of delayed transfer of critically ill stroke patients from the emergency department to the Neuro-ICU. Neurocrit Care 2010;13:75-81.

17. Van Beijnum J, Lovelock CE, Cordonnier C, et al. Outcome after spontaneous and arteriovenous malformation-related intracerebral haemorrhage: population-based studies. Brain 2009;132:537-43.

18. Hauerberg J, Eskesen V, Rosenørn J. The prognostic significance of intracerebral haematoma as shown on CT scanning after aneurysmal subarachnoid haemorrhage. $\mathrm{Br} J$ Neurosurg 1994;8:333-9.

19. Cordonnier C, Klijn CJM, van Beijnum J, et al. Radiological investigation of spontaneous intracerebral hemorrhage: systematic review and trinational survey. Stroke 2010;41:685-90.

20. Patel HC, Bouamra O, Woodford M, et al. Clinical article: mortality associated with severe head injury in the elderly. Acta Neurochir (Wien) 2010;152:1353-7; discussion 1357.

21. Bateman BT, Pile-Spellman J, Gutin PH, et al. Meningioma resection in the elderly: nationwide inpatient sample, 1998-2002. Neurosurgery 2005;57:866-72; discussion 866-872.

22. Lacroix M, Abi-Said D, Fourney DR, et al. A multivariate analysis of 416 patients with glioblastoma multiforme: prognosis, extent of resection, and survival. J Neurosurg 2001;95:190-8.

23. Linn FHH, Rinkel GJE, Algra A, et al. Incidence of subarachnoid hemorrhage: role of region, year, and rate of computed tomography: a meta-analysis. Stroke 1996;27:625-9.

24. Hemphill JC III, Newman J, Zhao S, et al. Hospital usage of early do-not-resuscitate orders and outcome after intracerebral hemorrhage. Stroke 2004;35:1130-4.

25. Morgenstern LB, Hemphill JC, Anderson C, et al. Guidelines for the management of spontaneous intracerebral hemorrhage: a guideline for healthcare professionals from the American Heart Association/American Stroke Association. Stroke 2010;41: 2108-29.

26. Naff N, Williams MA, Keyl PM, et al. Low-dose recombinant tissue-type plasminogen activator enhances clot resolution in brain hemorrhage. The intraventricular hemorrhage thrombolysis trial. Stroke 2011;42:3009-16. 